Regards sur l'économie allemande

Bulletin économique du CIRAC

$68 \mid 2004$

Varia

\title{
Volkswagen : programme en 7 points de Peter
}

Hartz

Isabelle Bourgeois

\section{(2) OpenEdition}

\section{Journals}

Édition électronique

URL : http://journals.openedition.org/rea/3634

DOI : $10.4000 /$ rea.3634

ISBN : 978-2-8218-0832-4

ISSN : 1965-0787

\section{Éditeur}

CIRAC

\section{Édition imprimée}

Date de publication : 1 octobre 2004

Pagination : 34-35

ISSN : 1156-8992

\section{Référence électronique}

Isabelle Bourgeois, « Volkswagen : programme en 7 points de Peter Hartz », Regards sur l'économie allemande [En ligne], 68 | octobre 2004, mis en ligne le 29 avril 2009, consulté le 15 septembre 2020 URL : http://journals.openedition.org/rea/3634

Ce document a été généré automatiquement le 15 septembre 2020

(c) CIRAC 


\title{
Volkswagen : programme en 7 points de Peter Hartz
}

\author{
Isabelle Bourgeois
}

1 Les négociations salariales ouvertes chez Volkswagen (siège : Wolfsburg, Basse-Saxe), le 15-06-2004 et qui doivent prendre fin normalement le 27-10-2004, jour de l'expiration de la convention précédente, s'effectuent dans un contexte particulier : après Siemens et DaimlerChrysler (voir dans ce numéro), le groupe VW mettra-t-il fin lui aussi aux 35 heures? Le constructeur, qui voit se réduire ses parts de marché mondiales dans le contexte de la crise automobile actuelle et voit ralentir ses ventes même en Allemagne, est contraint de réaliser des économies drastiques.

\section{VW doit comprimer de $30 \%$ sa masse salariale, ...}

2 VW a donc annoncé à la fin de l'été son intention de réduire de $30 \%$ d'ici 2011 sa masse salariale dans ses six sites ouest-allemands (effectif : 103000 salariés sur un total en Allemagne de 176544 ) pour la ramener à terme de 6,8 milliards $€$ à 4,8 milliards $€$. Pour y parvenir, il n'a que deux solutions: le gel des salaires ou la suppression de 30000 emplois. Il est vrai que le facteur travail est nettement plus cher chez VW qu'ailleurs : les salaires y sont supérieurs de $20 \%$ aux normes fixées pour l'ensemble de la branche à l'ouest par les conventions collectives. Chez VW, en effet, où le taux de syndicalisation est de $90 \%$, conditions de travail et de rémunération sont régies par une « convention-maison " particulièrement généreuse, et qui vaut à VW la réputation de « laboratoire social».

\section{... IG Metall revendique $4 \%$ de hausse salariale}

Or dans le cadre des négociations salariales de cet automne, IG Metall revendique une hausse de $4 \%$. Une revendication considérée comme indéfendable par la direction, et notamment par Peter Hartz, membre du directoire, directeur des affaires sociales (Arbeitsdirektor). Dès avant l'ouverture officielle des négociations, il expliquait la 
situation dans une conférence de presse du 23-08-04: "les temps ont changé... il n'y a aucune marge de manœuvre pour une augmentation ".

\section{Peter Hartz propose un compromis en 7 points :}

4 En guise d'ouverture permettant de concilier les intérêts des salariés et du groupe, Peter Hartz - le même que celui qui avait présidé la Commission du même nom, instituée par le chancelier Schröder pour élaborer les réformes du marché de l'emploi a soumis aux membres de la commission chargés de renégocier la convention salariale une liste de propositions, intitulée: « 7 points pour une garantie de l'emploi». Détaillées avec un grand sens de la pédagogie, elles visent toutes une normalisation à terme du niveau des salaires, obtenue grâce à une modulation particulièrement innovante du temps de travail et des rémunérations.

Résumé des « 7 points pour la garantie de l'emploi » de Peter Hartz :

... prime au résultat...

1. "Politique salariale ". VW se refuse à toute hausse des salaires, mais préconise d'accroître la part de l'intéressement, qui pourrait à terme atteindre $30 \%$.

2. "Job-Familien ». Il convient de « simplifier radicalement » la grille unique de rémunération du groupe (actuellement: 22 échelons pour plus de 4200 fonctions). Le nombre d'échelons devrait être ramené à 12 , et les diverses fonctions rassemblées en "familles" (Job-Familien). Cette nouvelle grille, plus souple, devrait entrer en vigueur dès 2005.

... modulation du temps de travail..

3. «Flexibilité ". Le système des comptes épargne temps devra être développé avec, à terme, un corridor de 區 400 heures. Cela assurerait une plus grande réactivité aux fluctuations du marché tout en permettant de supprimer les primes d'heures supplémentaires. De telles primes ne seraient plus versées qu'au-delà d'une durée hebdomadaire moyenne de travail de 40 heures.

4. « Temps de travail ». Adoption du principe : « les jeunes travaillent plus longtemps, les plus âgés, moins longtemps ». Grâce aux comptes épargnetemps, les salariés âgés pourraient continuer à bénéficier de la retraite anticipée.

... et des rémunérations...

5. « Module santé ». VW pourrait faire bénéficier ses salariés de prestations spécifiques, comme l'affiliation à des caisses d'assurance complémentaires pour couvrir par exemple les coûts des prothèses dentaires. Les salariés rembourseraient ces prestations santé par un forfait d'heures supplémentaires ou en finançant eux-mêmes une partie des prestations complémentaires que prévoit la convention de branche (formation continue, par ex.).

... mise en concurrence des sites ...

6. "Co-investissement pour la garantie de l'emploi ». Les divers sites de VW devraient avoir une plus grande indépendance et être mis en concurrence lors des choix stratégiques d'investissement effectués au niveau $\mathrm{du}$ groupe. La concurrence porterait sur les avantages compétitifs respectifs. Pour être élu (attirer les investissements), le site pourrait décider par exemple d'allonger la durée du travail pendant quelque temps - ce serait sa contribution au financement de l'investissement (co-investissement).

... la fin de l'emploi à vie chez VW 
7. «Une formation compétitive ». Les salaires des apprentis devraient, comme tous les autres, être ramenés progressivement au niveau de la norme de branche (soit $20 \%$ de moins). La différence ainsi économisée devrait être investie dans la création de places d'apprentissage supplémentaires. L'embauche ne devrait plus être automatique à l'issue de la formation, mais s'effectuer en fonction des besoins réels du groupe comme de la compétence/performance de l'apprenti.

\section{La fin programmée de l'exception Volkswagen...}

5 Ces sept points ne seront pas tous adoptés en l'état, mais ils inspireront très largement la teneur de l'accord à venir. Ils s'inscrivent en effet dans le prolongement des accords conclus chez Siemens ou DaimlerChrysler (réduction des coûts salariaux grâce à un allongement de la durée du temps de travail, rémunération modulable avec prime au résultat). Et ils vont plus loin, prenant en considération la problématique de la pénibilité de certaines tâches pour les salariés âgés dans l'industrie. Enfin, ils replacent la question de la détermination des salaires dans leur contexte: le système de protection sociale et l'avenir de son financement (la question de la prise en charge des prothèses dentaires est débattue dans le cadre de la poursuite de la réforme de l'assurance maladie) comme la question des qualifications des jeunes ou de la pénurie de places d'apprentissage (voir dans ce numéro).

\section{... en écho à la réforme de l'Etat social allemand}

6 Ces propositions, formulées par un membre du directoire, rappelons-le, sont également révélatrices de l'état des réflexions sur la restructuration du groupe VW et la modernisation de sa culture sociale. Le fait qu'elles émanent de Peter Hartz leur confère un surcroît de valeur symbolique. Car bien qu'il s'agisse d'un cas particulier du fait des généreuses dérogations à la norme de branche, la délicate réforme du « modèle VW » qui s'engage aujourd'hui est le pendant, au niveau d'une entreprise-phare de la culture et de l'histoire sociales allemandes, des grandes réformes d'un « modèle social » allemand qui, à l'avenir, devra trouver de nouveaux équilibres. (IB)

\section{INDEX}

Mots-clés : Volkswagen, industrie automobile, syndicat, réforme, Hartz, revenu, salaire, emploi 